\title{
Shared Experience Building Around the Family Crucible of Cancer
}

\author{
W. David Robinson \\ University of Nebraska Medical Center \\ Jason S. Carroll \\ Brigham Young University - Provo, jcarroll@byu.edu \\ Wendy L. Watson \\ Brigham Young University - Provo
}

Follow this and additional works at: https://scholarsarchive.byu.edu/facpub

Part of the Other Social and Behavioral Sciences Commons

\section{Original Publication Citation}

Robinson, W. D.,Carroll, J. S., \& Watson, W. L. (2005). Shared Experience Building Around the Family Crucible of Cancer. Families, Systems, and Health, 23, 131-147.

\section{BYU ScholarsArchive Citation}

Robinson, W. David; Carroll, Jason S.; and Watson, Wendy L., "Shared Experience Building Around the Family Crucible of Cancer" (2005). Faculty Publications. 4334.

https://scholarsarchive.byu.edu/facpub/4334 


\section{Shared Experience Building Around the Family Crucible of Cancer}

W. David Robinson, PhD

JASON S. CARROLL, PHD

Wendy L. Watson, PhD

This study was designed to gain a greater understanding of the effects cancer has on families while simultaneously developing family therapy interventions that are helpful for families experiencing the crucible of cancer. On the basis of an action research paradigm, the authors designed an intensive case-analysis protocol involving reflective interviewing to learn about the personal perspectives of family members who participate in therapeutic treatment for cancer. An integration of reflective insight by the families, their therapist, and the research team ultimately revealed that cancer families face an ongoing struggle (a) between feelings of isolation and connected-

W. David Robinson, PhD, Department of Family Medicine, University of Nebraska Medical Center; Jason S. Carroll, PhD, School of Family Life, Brigham Young University; Wendy L. Watson, PhD, Marriage and Family Therapy Program, Brigham Young University.

The research began when W. David Robinson, $\mathrm{PhD}$, was a graduate student at Brigham Young University.

We thank Jennifer Creer, MA, for her help in preparing this article. We especially want to thank the participant families for their willingness to open up their lives and share their experiences with us

Their desire was that others would benefit from their lived experiences.

Correspondence concerning this article should be addressed to W. David Robinson, PhD, Department of Family Medicine, University of Nebraska Medical Center, 983075 Nebraska Medical Center, Omaha, NE 68198-3075. E-mail: wdrobins@unmc.edu ness, (b) to make meaning of cancer in their life, and (c) to find ways of incorporating cancer into their current world view. The study also revealed shared experience building and interactive psychoeducation to be the most significant components of the therapeutic treatment process for cancer families.

$\mathrm{C}$ ancer profoundly intrudes on the body, the individual, and the family. Some scholars have referred to illnesses such as cancer as "emotional crucibles," referring to how the illness creates an "emotional roller-coaster ride that is both draining and empowering" for families (McDaniel, Hepworth, \& Doherty, 1997, p. 3). Despite the prevalence and impact of cancer, information on clinical interventions for families in which a member copes with cancer is only beginning to emerge. There have been some documented efforts to offer psychosocial interventions to cancer patients (Chollar, 1994; Cunningham, 1992; Spiegel, 1990) or to other family members (Northhouse, 1996; Ostroff \& Steinglass, 1996; Scott et al., 2003), but seldom does the treatment focus on the family unit as a whole.

The literature particularly offers little information regarding the subjective, personal perspectives of family members who participate in therapeutic treatment, de- 
spite the fact that several qualitative studies have shown that family support is a key factor in improving psychosocial functioning of cancer patients (Lewis, Hammond, \& Woods, 1993) and that family functioning may be more important than other forms of support (Cooley \& Moriarty, 1997). Furthermore, scholars have concluded that more research "based on family developmental and systems theory is needed" (Kristjanson \& Ashcroft, 1994, p. 3). Documenting the lived experiences of cancer families who participate in psychotherapy provides unique phenomenological insight that is essential to the development of these models.

\section{PURPOSE OF THE STUDY}

This study was designed to answer the following overarching research question: How can marriage and family therapists and other healthcare professionals most effectively assist families experiencing the crucible of cancer? Specifically, the goals are (a) to gain a greater understanding of the effects cancer has on family development and functioning; (b) to develop specific, tailored family therapy interventions that help the families participating in the project; and (c) to gain insight that can guide the development of general intervention strategies that may be useful for clinical work with other families experiencing cancer. This dualistic research/treatment approach ultimately contributes to a greater understanding of families' lived experiences with cancer and their perceptions of the therapeutic process.

\section{CANCER IN THE FAMILY}

Because of the open-ended, action research paradigm that guides this research/ intervention project, both the research and the therapist teams relied heavily on previous research to prepare for the collaborative interaction with families (Baider, Cooper, \& De-Nour, 1996). The literature review led the research team to become particularly familiar with the illness tra- jectory of cancer, common family responses to cancer, the role of healthcare providers, and previous psychotherapeutic interventions used with families coping with cancer.

\section{Cancer Illness Trajectory}

The degree of limitation imposed by the illness and the outlook for survival give some insight into the possible problems that the family could be experiencing. Rolland (1994) charted the psychosocial trajectory of chronic illnesses such as cancer by separating the disease experience into three phases. During the crisis phase, the family must decide what cancer means to them; this period requires role changes and shifts in patterned parts of family life. The chronic phase, often called the "long haul," is the process during which the family is coming to grips with the illness and, often, caregiver burnout. The terminal phase includes the periods of mourning, bereavement, and resolution of loss. By understanding the illness trajectory and its developmental stages, the research and therapy teams acquired an idea of possible issues the families might encounter and were better able to evaluate the effects of the illness on the family.

\section{Family Responses to Cancer}

Wright, Watson, and Bell (1996) asserted that "how families adapt, manage, and cope with illness arises from their beliefs about the illness confronting them" (p. 23). Similarly, Gosselin (1989) found that difficulties often occur when family members hold too tightly to existing beliefs and do not allow new information to change their outlook or perspective. Therefore, by focusing on the beliefs a family has about cancer, clinicians are better able to understand the current context of the family and the impact the illness is having in the multiple domains of family life. Temoshok and Dreher (1992) found that individuals in families responding to cancer cope better if they have a supportive psychosocial network, are hopeful, and have a sense of per- 
sonal control. Cancer patient caregivers are also subject to a large amount of stress; a significant minority of individuals in this role develop emotional distress or psychiatric comorbidity. Pitceathly and Maguire (2003) suggested that although a variety of interventions for caregivers have been reported, interventions are needed that target high-risk cancers and are designed to bring about psychological and emotional gains. Scott et al. (2003) also found that communication about their family member's cancer may reduce psychological, social, and sibling problems for children and adolescents.

\section{Role of Healthcare Providers}

Because families experiencing cancer are intimately involved in the healthcare system, it is also important to understand how the relationship between families and healthcare professionals can be improved. Several studies have revealed that straightforward, honest communication between families and physicians facilitates and improves this intimate relationship (Hull, 1989; Kristjanson, 1989). Further, Stedeford (1981) determined that poor communication between health professionals and families causes cancer patients and their families more suffering than any other factor except unrelieved pain. Various studies indicate that effective physician-patient treatment improves patient satisfaction and outcomes (Levinson \& Roter, 1995; Ong, Visser, Lammes, \& Haes, 2000; Seaburn, Lorenz, Gunn, Gawinski, \& Mauksch, 1996). Families able to evaluate their roles in managing the medical care aspects of cancer are better advocates for themselves.

\section{Previous Psychotherapeutic Interventions}

The literature reviewed revealed that group therapy strategies, the mind and body connection, and identification of the family belief paradigm have contributed to past therapeutic approaches. Group therapy strategies have been used to help can- cer patients cope with their illness (Cunningham, 1992; Reele, 1994). Galinsky (1985) identified the following five objectives for group therapy with cancer patients: (a) provide social support, (b) share feelings and concerns, (c) develop coping skills, (d) gather information and education, and (e) consider existential issues. Spiegel, Bloom, Kraemer, and Gottheil (1989) found that metastatic breast cancer patients who received group psychotherapy lived almost twice as long as those without therapeutic intervention.

An emerging body of evidence also suggests that therapeutic interventions for families experiencing illnesses such as cancer should be based on a paradigm that recognizes the bidirectional connection between mind and body (Spiegel, 1990). Spiegel (1990) explained that comprehensive cancer treatment involves both medical (surgery, chemotherapy, etc.) treatment and psychosocial support. Cunningham (1992) used a teeter-totter analogy to explain that psychological help in conjunction with body-healing mechanisms and medical treatment are used to counterbalance, or outweigh, the effects of cancer on the body. He conceptually divided the therapeutic journey into three phases: (a) taking control, which involves learning to have some control over reactions to one's environment, both mentally and physically; (b) getting connected, which involves the understanding of and effort to connect with the universe, a spiritual being, and humankind; and (c) facilitating a search for meaning regarding all aspects of living.

Medical family therapists also recognize that for an intervention to be effective there must be a "fit between the therapeutic offerings of the clinician and the biopsychosocial-spiritual structures of family members" (Wright et al., 1996, p. 87). By working within a family's paradigm, therapists are better positioned to help family members change constraining beliefs, thus allowing the meaning of the cancer to evolve. Through this process, emotional en- 
ergy is freed up, and the family is able to use its strengths to counter some of the negative effects of the illness (Gosselin, 1989; Wright et al., 1996). For example, Sellers (2000) researched the efficacy of a biopsychosocial collaborative treatment model for cancer patients and their families by adding a medical family therapist to the oncology practice. After completion of the study, patient surveys reported that $90 \%$ of patients experienced a significant decrease in pain and suffering after the work they did with the medical family therapist; $91 \%$ of patients learned to incorporate psychosocial resources such as friends, family, prayer, and bravery into their treatment; and $73 \%$ of patients learned to develop and maintain hope as well as to plan for approaching problems they may face during treatment.

The information regarding the families' responses to cancer, the illness trajectory, the role of healthcare providers, and understanding the different psychotherapeutic approaches helped give clues to the basic areas that we investigated with the participant families. Because of our understanding of these major issues, we were able to more effectively conduct the research aspect of this project.

\section{STUDY DESIGN AND METHOD}

\section{Research Approach}

We chose action research as our guiding paradigm to answer the following question: How can marriage and family therapists most effectively help families experiencing the crucible of cancer? Specifically, we designed a case-analysis protocol that used reflective interviewing procedures to solicit the participant families' ongoing feedback and insight throughout the therapy and research process. Action research enables the participants to collaborate as coresearchers in finding ways to influence their own situations through inquiry, action, and reflection (Holter \& Schwartz-Barcott, 1993; Reason, 1994). Our hope was that this type of self- reflective, case-analytic approach would help "bridge the gap between theory, research, and practice and incorporate both humanistic and naturalistic scientific methods" (Holter \& Schwartz-Barcott, 1993, p. 298).

Through the use of an action research design, the periodic progress of therapy, or lack thereof, can be investigated, and adjustments can be made during the research process. Reflexivity (the process of reviewing what is being done) is introduced into the research process through an open dialogue among the families, the therapist, and the primary researchers. In this process, the "research-practitioner evaluates the situation, develops a theory to account for that situation, tests the theory by constructing and implementing a clinical intervention, evaluates the new situation, modifies the theory accordingly, and so on in a continuous cycle or spiral" (Rolfe, 1996, p. 1317).

Holter and Schwartz-Barcott (1993) have identified four common characteristics of action research, which this study conforms to: (a) collaboration, (b) problem identification, (c) change in practice, and (d) theory development. Collaboration with the families around the therapeutic process was continued throughout the family therapy intervention. The problem was identified by the participant families, and this information was used to verify or modify the focus of the intervention. Change in practice occurred on two levels, namely in the family and in the therapy. The therapist-family system challenged family practices that were counterproductive to effective coping. The process of therapy was continually evaluated and changed to best fit the specific needs of the family. Theory development occurred as the therapistfamily researcher system challenged the functionality of the proposed and implemented interventions.

In our review of the literature, we identified two studies that used action research-based approaches to investigate how families adapt to cancer. Norman and 
Brandeis (1992) assisted cancer survivors by including them in the research process, which developed effective programs for cancer survivors and overcame potential service barriers. They found that bone marrow transplant survivors' major concerns were general physical health, relationships with family, child bearing, health insurance, dealing with feelings about being a survivor, and planning for the future. A major change in the treatment approach was to create discussion groups for the survivors. Chesler and Chesney (1995) also used action research to develop self-help groups for parents of children with cancer. The groups identified the following needs: (a) consulting to deal with trauma, (b) leadership training, (c) guidelines for starting groups, (d) advocacy training to help families work with medical staff, (e) mediation between families and medical staff, (e) disseminating findings, and $(\mathrm{g})$ influencing a medical facility's policy on group function.

The research team for this study was instructed on the importance of critical subjectivity, meaning that the coresearchers do not suppress their own experiences but work to see through the subjective perceptions and possible distortions. They are not naive subjects but actually have an important role to monitor themselves and the research process and come to better understand themselves (Reason, 1996). Within this type of paradigm, the purpose of human inquiry is not to find truth but to heal-to make whole (Reason, 1996).

\section{Participants}

Because action research requires active participation, the participant families were chosen, through an initial screening interview, for their ability to express themselves and willingness to participate. One adult family member must have been formally diagnosed with the chronic stage of cancer, defined as having completed treatment, but not be terminally ill. Several participant families for this study were identified through references from a medical family therapist and a local ecclesiastical leader, and 4 were interviewed as potential candidates. Two of these families were selected, the Holbrook and Miller families (names and distinguishing details of the families have been changed). The sample was purposely limited to 2 families because of the intensive procedures involved.

\section{The Holbrook Family}

When asked to describe themselves, the Holbrook family used the following statement: "We are a self-reliant, work-together family who knows how to depend on one another." Bill (the father, in his early $60 \mathrm{~s}$ and a cancer patient) and Beth (the mother, in her early 60s) were the key participants from this family. Married for over 35 years, they have five children, ranging in age from 20 to the late 30 s. Four of the children agreed to explore their experience with their father's cancer. Bill was diagnosed with prostate cancer 1 year prior to the study. He and Beth spent the next several months researching different treatment options and decided to use a new technique in which doctors implant a number of radioactive seeds directly into the prostate. Just before the study, he found out that the treatment was working. The family had received no psychotherapy before their inclusion in the study.

\section{The Miller Family}

When asked to describe themselves, the Miller family used the following statement: "We are a loving family who strives to do what is right. We demand kindness and love of ourselves. We believe that character development is an important goal for each of us." This family consists of John (the father, in his late 40s), Susan (the mother, in her late $40 \mathrm{~s}$ and a cancer patient), Rachel (a teenager, Susan's daughter from a previous marriage), Zachary (the 6-yearold biological son of John and Susan), and Celia (John and Susan's 2-year-old daughter). All family members except Celia participated at some point in the sessions. 
John and Susan had been married more than 7 years.

Susan was diagnosed with advanced breast cancer approximately 1 year before beginning the study. Her treatments consisted of a lumpectomy, a single mastectomy, and 9 months of a combination of chemotherapy and radiation. She was told that she had approximately an $80 \%$ chance of reoccurrence. The family received no psychotherapy for their experience with cancer prior to this study, except for a few sessions of sand-tray therapy.

\section{Procedures}

The project team consisted of three primary subteams: the research team (consisting of three therapist-scholars), the therapist team (consisting of a doctoral-internlevel medical family therapist), and the family team (consisting of the cancer patient and his or her participating family members). The metaprocess of the project involved five components: (a) a recruitment and protocol meeting, (b) a series of weekly therapy and research sessions, (c) a midtherapy research meeting, (d) another series of weekly therapy and research sessions, and (e) a final wrap-up session (see Figure 1 for an illustration of the action research-therapy cycle). Each of these steps is described briefly below.

\section{Recruitment and Protocol Meeting}

An initial meeting introduced the families to the therapist and research teams to orient the families to the general purposes of the study, to review the basic protocol, and to answer any questions they might have.

\section{Weekly Therapy/Research Meetings}

Each of the families completed between 6 and 12 sessions of weekly meetings with the professional teams, which were videotaped in their entirety; detailed process notes were taken. Following each meeting, written transcripts were made and distributed to the participants to refresh their memories of the previous session and help them identify possible steps for the next sessions. Each weekly meeting consisted of a therapy and a research session.

The families met with the therapist for a session, typically 60 to $90 \mathrm{~min}$ in length and observed by the research team, who, as needed, provided observations, ideas, and questions to the therapy group during the session. Directly after the therapy session, the full team (e.g., family, therapist, and
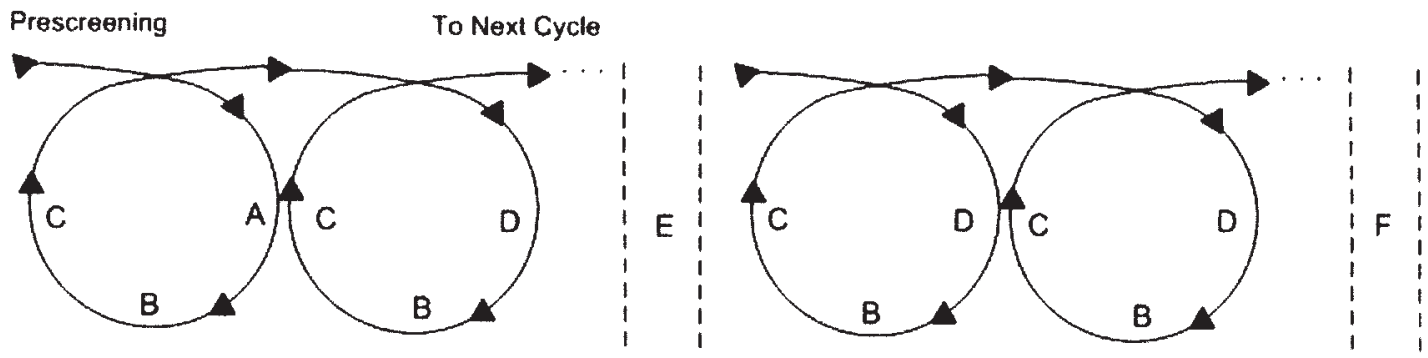

Figure 1. Action research-therapy cycle. The goals of this cycle are to (a) help participant families better cope with cancer and (b) develop strategies to help other cancer-afflicted families. $\mathrm{A}=$ therapy (assessment and intervention); $\mathrm{B}=$ research session (investigate, reflect, and plan); $\mathrm{C}=$ action (assignments for the family, coevolve the next session); $\mathrm{D}=$ therapy (modified by coevolved planning session); $\mathrm{E}=$ midtherapy research session; $\mathrm{F}=$ final wrap-up session. 
research team) held a research session, typically 20 to $60 \mathrm{~min}$ in length. To allow the participants to fully participate in a researcher role, a break was given, the room was rearranged, and the researchers entered the room. The primary researcher facilitated all research sessions.

\section{Midtherapy Research Session}

At the midpoint of therapy, a 90-min working meeting was held, involving the project team to identify strengths, effectiveness, weaknesses, and future direction of therapy. We also discussed the larger trajectory of the therapy process to that point and large metaissues pertaining to the effectiveness of the current direction. Families were presented with some preliminary analyses by the research team (member checked), and plans were made for the next series of weekly sessions.

\section{Second Group of Weekly Therapy/Research Meetings}

Following the midtherapy session, each family participated in three to six more weekly therapy/research sessions, which followed a similar protocol as described above.

\section{Final Wrap-Up Session}

The capstone meeting in the collaborative process was a final, 2 -hr wrap-up session to reflect on what the families learned in the areas of living with cancer, the therapy process, and their reflections of participating in a reflective research project.

\section{Data Analysis}

Initially, preliminary data analysis occurred during each of the research sessions, with the family, therapist, and researchers analyzing the effectiveness of the therapy sessions. All project members were invited to share key insights from the day's work together, which were incorporated into subsequent sessions and catalogued for later analyses. On the completion of the therapy process, final analyses were con- ducted on the transcribed data from all of the therapy/research sessions. The data were analyzed according to a standard grounded theory approach (Strauss \& Corbin, 1998), which included a five-stage analysis process. In the first stage, the members of the research team independently analyzed the transcripts and used open coding techniques to categorize the data. The second stage involved axial coding, in which each researcher individually organized the data into identified common themes. The third stage involved a group meeting among the researchers that involved a discussion of major identified themes, unanimously agreed on for study inclusion. In the fourth stage, the researchers selectively coded, or categorized, themes into major and minor groupings that were used to understand the proposed therapeutic process and to develop a theory of family therapy. Results were again member checked with the families to ensure reliability and to verify the initial analyses (Guba \& Lincoln, 1989).

\section{RESULTS}

We have chosen to separate the findings into two main categories: (a) families' experiences with cancer, and (b) the therapeutic process around the crucible of cancer. We focus our attention on the lived experience of cancer and the struggles of the participant families. Our analyses reveal three overarching themes: (a) the balance between isolation and connection, (b) beliefs about the meaning of life and death, and (c) finding balance between old and new world views. Our evaluation of the coevolved interventions used in the study reveals several important components of the therapeutic process, including assessment, treatment strategies, and the structure of therapy.

\section{Family Experiences With Cancer}

\section{Isolation Versus Connection}

The ongoing tension between isolation and connection was the core process in the 
family members' experiences around cancer. These emotional polarities represent the individuals' and families' struggles to adequately receive support from one another and outsiders, even though the cancer experience is often an isolating experience. It was clear from the transcripts that a sense of isolation was a very common and difficult part of the cancer experience for most of the participants. In most instances, feelings of aloneness emerged from a perceived lack of support from loved ones and caregivers. However, for 2 of the participants, their feelings of isolation were more about facing a potential death, knowing that they have to travel at least part of the road alone. Susan, one of the cancer patients, stated,

I remember sitting there with the chemotherapy going in me and John [her husband] sitting next to me, and I think you [turning to John] were asleep, and I remember at that moment thinking-you know, there is a very clear reality that we are alone in this world ... and feeling all of this comfort from other people. But there are things that we do in our lives that are $a$-lone! I don't think I ever admitted that before or ever saw it as clearly.... It was a solitary piece to a journey.

Another central aspect of the dialectical tension between isolation and connection involved social interactions in which people were clearly uncomfortable with the illness and uncertain how to support the family. Some people deal with this discomfort by avoiding contact with the cancer patient altogether-often intensifying the sense of isolation. Another cancer patient, Bill, stated,

All of a sudden you are really kind of isolated. People don't like talking about cancer. ... They were very uncomfortable. It is like the plague or something, you know. It is like if they touch you they are going to get it. I mean, they don't say that-but people are kind of that way. They don't want to hurt your feelings.

Sometimes the cancer patient and other family members attributed discomfort in others as a "pulling away" based on a fear of contagion. Fostering a sense of how to handle these difficult situations is extremely important, because avoidance exacerbates pain and distress for cancer patients. Our team found that this could be remedied through fostering a nonanxious presence that communicates, "I am here for you, whatever you need."

It is interesting that both of the participant families also expressed that the cancer had fostered moments of intense connection as they were drawn to each other in very powerful ways. Their extended families and outside-family support were crucial to their coping. This type of support was reported to be particularly helpful in reducing the caregiver's burden.

\section{Beliefs About the Purpose of Life and Death}

The second major theme was the role beliefs about the purpose of life and death played in the families' therapeutic journey. Three emotional polarities emerged in the families' experiences: (a) senselessness versus meaning, (b) loss versus renewal, and (c) essential versus important.

Senselessness versus meaning: $A$ search for purpose. Families experiencing cancer often make sense of the illness by finding a spiritual meaning in suffering (HalonaTrujillo, 1998; McDaniel et al., 1997). John (husband of patient) stated, "The most important thing is that people have got to have a spiritual relationship. I don't see how people can go through cancer ... [or] go through life without a spiritual relationship ... it gives life meaning."

Senselessness, conversely, illustrates the absence of meaning-cancer is seen as void of purpose, and nothing can be learned from it. Meaning and senselessness are 
emotional polarities, as participants from both families illustrated. Susan (patient) stated,

Maybe that's part of the defense [choked up] of having had to go through that [referring to the cancer]. By darn, there has to be something in this that is good, I'm not going to suffer through this and beyond what I could even comprehend, the suffering, and not come out with something. You guys [referring to her husband and daughter] are, "By darn we didn't want this!" and I'm on the other end, "By darn we've got it and we're going to make something great out of this!"

In contrast, Susan's daughter Rachel, when speaking about her experience with her mother's cancer, stated, "I know that you guys [referring to her parents] think that there's a lot of good things, but I really have a hard time thinking that there is anything good." This roller-coaster ride of emotions-vacillating between a sense of meaning and senselessness-is a normal reaction to a very difficult situation.

Loss versus renewal: Coming to terms with a new life. With cancer there is nearly always loss, ranging from loss of dreams and abilities to loss of life. Participants in both families illustrated this. Bill (cancer patient) stated, "And then you got the other pressures that are on because of what happens. The physical response to that is loss of urinary functions, loss of bowel, loss of sexuality, those things are all there looming."

Susan (cancer patient) stated,

Will I be alive to be a mother? Are they gonna lose their mother? I think, yeah, I looked at John and said, just after we heard about it, we hadn't really made the decision but we both knew where our hearts were going and I said, "We're never going to Spain, are we?" You know there's a sacrifice of time for he and I to just play out the last portion of our life, you know.

Renewal often comes after loss, perhaps in part from an improvement in physical health, but it may also come from family efforts to regain the things that cancer has taken. Susan stated, "I kept thinking, it seems that month to month a little bit more of me comes back. So lately I've been starting to think that the kind of light and fun person inside me will resurface again." The experience of regaining and reclaiming losses can be very liberating and positive, as families are able to have life-affirming experiences that are directly connected to having faced the losses associated with serious illness.

Essential versus important: The family's efforts to reorganize their life. This process centered on finding a balance between the "essential" (as seen through the lens of cancer) and the mundane. This theme of essential versus important can be seen in different ways (e.g., short term vs. long term) and was found to be important in both families. Specifically, they reprioritized their energy and time toward what they deemed the "essential aspects of life," such as children and other family relationships. Bill stated,

We do things more. I appreciate her [turning to his wife] more now... . I hate to say you ever take your wife for granted, but you kind of do. She is there and she is going to do things and I would rather spend more time with her now ... being with her is more essential. ... That is a change that I have seen.

This shift toward "the essential" was a common point of contention with healthy family members who recognized the necessity of continuing the mundane yet important tasks, such as housework, jobs, and bills. A central aspect of the therapy process for both families involved communicating about incongruence and finding a 
balance that honored the cancer patient's new emphasis on "essential projects" while simultaneously developing patterns that would also take care of the tasks necessary to maintain a home and family relationships. For example, John (husband of cancer patient) stated,

It's like the day-to-day things that are necessary in a family relationship or family things to do and the yearly things are done like our taxes and stuff like that are always put off because that is a struggle to work through. But it's more important for her at the moment to build a strong relationship with Celia and Zachary, especially how young they are, and with Rachel because she may not be here in a year or five years.... And so in that sense those short-term things are really vital and important.

\section{Old World Versus New World}

The final theme found in this study was finding a balance between the old world of health versus the new world of cancer. Families are usually unprepared for the changes cancer brings and often find that it creates a new world for them (Cohen, 1993;
Robinson \& Smith, 1998), which can be compared with entering a new, uncharted land (Shuman, 1996). Like immigrants in a new country, families begin the process of either accepting and creating a life in this new world or taking extreme efforts to keep life as it used to be (Robinson \& Smith, 1998). Because cancer inevitably changes life, families need to learn how to move forward and develop a life that includes the cancer component (see Figure 2).

Maintaining the old world view. It was clear that some members of both families viewed "getting better" as resuming life as it was before the cancer was discovered. These individuals expressed frustration when others wanted to discuss and recognize the role cancer played in their families now. In short, they were trying to continue to live life as if nothing had happened. Susan's daughter, Rachel, said,

I just want to get past it. I think that I'm fine and I really don't want to be bringing it up all the time because it's just not one of those things that you want to have, you know, it's not like dinner table discussion. . . . It feels like that is just something that you leave closed and then if there's something

\section{Old World vs. New World Views}

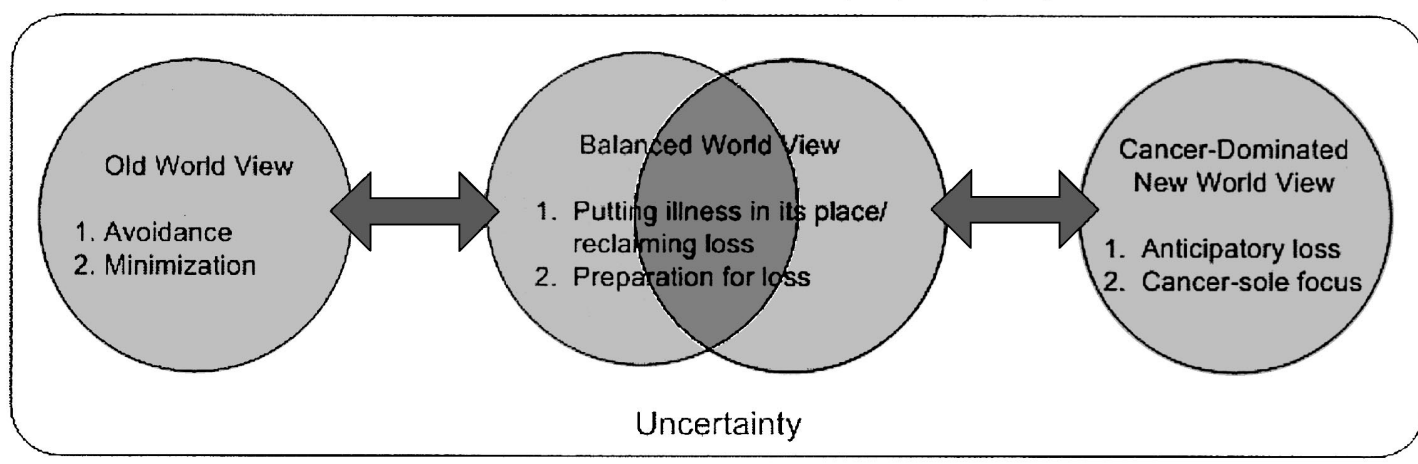

Belief About the Probability of Death Occuring

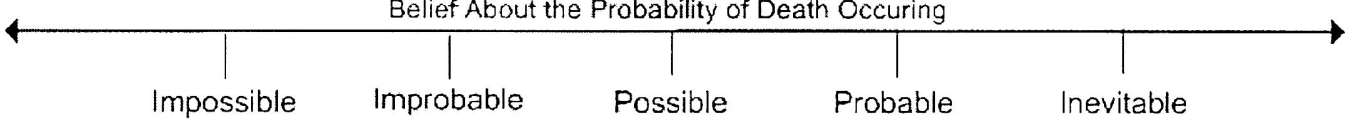

Figure 2. Old world versus new world views. 
that really needs to be talked about then you talk about it. ... Revisiting it all the time is making it more and more like in the present tense.

An effort to live in the old world can limit the patient's ability to seek needed treatment, because treatment is not a part of the previously recognized patterns. Efforts to avoid and/or minimize the cancer or its effects on the family seem to be prevalent in this world view as well. It is critical that death be addressed, or preparations, such as putting finances in order, preparing a will, or saying goodbye, may never occur. At times these views can reach an extreme at which family members are unable and even forbidden to talk about the illness and the possibility of death. This fosters feelings of isolation and may leave family members alone to deal with the grieving process when death eventually comes.

Aspects of cancer's illness trajectory may contribute to family members' desire to remain in the old world. By holding to the idea that cancer was in the past, several family members made attempts to live life as if nothing had happened. During the early parts of the chronic phase, many patients do not demonstrate noticeable signs of illness, and this allows many to stay in the old world. This is illustrated by Bill's (cancer patient) comments:

I can't speak for the others of course. But with prostate cancer, you are not sick. You know you are not physically ill. You don't. It is not like you are having the flu. It is not like cutting your finger or your arm or breaking your arm and you go get it fixed and it hurts. You are forced to take some action. You know there is no forcing of action down on this acceptance. So it is easier to deny it.

Another characteristic of this paradigm is the belief that loss is impossible or improbable. This desire to live in the old world was found to be an important conceptual lens in understanding some of the attitudes and behaviors of the participating family members.

Living in a cancer-dominated new world view. The cancer-dominated new world view has its own challenges, because family members who completely accept the cancer and the prognosis oftentimes struggle with anticipatory loss (Becker, 1973; Lindemann, 1944; Rolland, 1994), which causes family members to pull away from one another in anticipation of death. Susan described this in reference to herself and her young daughter:

I know that, even when I think about Celia [2-year-old daughter], you know, in the daytime we used to play together.... As I look at my behavior with Celia, I've not withheld love and snuggles, but I've really withheld playtime so that she's not used to having that type of interaction.

When anticipatory loss begins to have this type of impact, family members begin prematurely to learn how to live without the cancer-afflicted member in their lives. Everything they do relates to cancer. This focus can cause the loss of hope in the future because all attention is on cancer.

Living in a balanced new world view: Learning to put illness in its place. It became clear in our work that progress was made when the families were able to develop a synthesis between the extremes of the old world and the cancer-dominated view of the new world. This involved creating appropriate space to discuss and manage the treatment aspects of the illness and to occasionally shut the cancer out. This balanced new world view encourages a stabilization of emotions, with some fluctuations, because individuals who dwell permanently in either of the extremes have a more difficult time dealing with cancer than those who find an appropriate degree of balance. One family described how they 
have learned to do this. Beth (wife of patient) stated,

When someone that you love an awful lot is in that situation, for me I just kind of compartmentalize that. You don't dwell on it. You realize it is there but you also realize that life has to go on and there are a lot of people depending on you and you just have to do what you have to do. And so you compartmentalize it. That is kind of, I deal with it as I have to deal with it, or as I need to deal with it, I will deal with it. And if you dwell on it, it destroys you.

The families agreed that the phrase "prepare as if this is your last day on earth, live as if you are going to live forever" captures much of the essence of the balanced world view. Many of the therapy processes centered on helping families find the balance between the old and new world views. The families gradually learned how to pull away from the extremes of the old and new world views. Uncertainty continued to be an issue for these individuals, and they learned to mediate it by "putting illness in its place" (Gonzalez, Steinglass, \& Reiss, 1989, p. 80). They were no longer willing to allow cancer to take over their life, nor were they willing to quietly wait and pretend that cancer did not have an influence on them. The following quote, taken from later in the therapy process, describes $\mathrm{Su}$ san's efforts to create a balanced new world view:

I said to the oncologist, "We want things back to normal." And he made an intellectual quip, "Well, what's normal anyway?" ... There was a "normal" that we had as a family. There were things that people could depend on, people depended on me. ... For me, I think part of it, I refuse to allow this disease ... to take the good things we have about life and about purpose and about family.... It's not going to take my attitude, the way we talk to each other, the way we treat each other. I have to stand up to it. Okay, you can have my breast, you can have a portion of my life, I don't know how much that will be. You can take me to my knees in agony, but you will not take these things. I will not allow that and I get to not allow it. It is the place I get to have power. It's the place that the cancer has to bow down and stop ... we have to have this attitude to remain close, not let it put us at odds or have bad memories or pessimism.... I won't surrender those things.

The balanced new world view controls illness by not allowing it to take over the family structure, rituals, and celebrations (Gonzalez et al., 1989; Steinglass, 1998). Cancer brings mortality into focus, yet family members in the balanced new world understand that loss is a possibility without being completely overwhelmed by its potential and are able to have hope.

The cancer experience creates many different circumstances. The way the family deals with these circumstances determines which "world" they are in. The old world and cancer-dominated worlds tended to create more problems and make the individual's or family's ability to cope with the stress of illness more complicated. The action research therapeutic process helped to identify the balanced new world view as another alternative that helped increase the family's and individual's ability to deal with the stresses that cancer imposed. Families' experiences with cancer, including isolation versus connection, beliefs about the purpose of life and death, and old world versus new world views, were important components in understanding the families' experience with cancer. Understanding these issues proved to be key to the therapeutic process. 


\section{Therapeutic Strategies for the Family Crucible of Cancer}

Family members were able to directly influence therapy through the reflective interviewing process. One major distinction that must be made is that family therapy with cancer patients (and perhaps those with other chronic illnesses) is not primarily about diagnosing and treating dysfunction but rather is intended to help families maintain or regain connection. This is unlike some of the medical and psychotherapeutic paradigms, which focus solely on individual dysfunction.

\section{Assessment-Understanding the Families' Experiences of Cancer}

The cycling processes of this study highlight the need for clinicians to use a multifaceted assessment protocol when working with families experiencing the crucible of cancer. This involves developing an indepth working knowledge of the individual and family experience with the illness through the following assessment strategies: (a) the collaborative development of a health and illness experience genogram, (b) the sharing of the family's illness stories through interview, (c) questions about the family's interaction with medical care workers, and (d) evaluating the need for targeted therapy sessions with different subsystems of the family (e.g., marital sessions, family sessions, parent-child sessions, or individual sessions).

\section{Treatment Strategies}

The process of shared experience building. A central assumption for our project team was that treatment for illness issues should be focused on creating family cohesion. The central therapy-related theme of this project was that therapy should be aimed at creating a forum that facilitates shared experience building (Wamboldt \& Reiss, 1989) among family members. Shared experience building-also known as developing shared meaning struc- tures-is based on developing a shared experience of healing, not necessarily a shared experience with cancer. In fact, our work together highlighted that recognizing and validating family members' different experiences with cancer was, paradoxically, one of the key components to creating a shared experience of healing. Although there may be elements of the cancer experience that are collectively shared among family members, it is common for each individual to have distinct ways of interpreting and experiencing the crucible.

Because of personal beliefs, previous life events, and a myriad of other reasons, each family member has parts of the experience that are unique. Herein lies a paradoxical component of families' experiences with cancer. As family members recognize and appreciate that their personal experiences differ, their collective experience becomes more shared. In other words, the validation of difference brings unity. Gilbert (1996) noted, "Rather than striving for a single view of the loss, or promoting a single style of grieving, family members need to come to recognize the similarities in their grieving and to reframe differences as strengths" (p. 279). When this is done, the cancer experience can be shared, even if family members maintain separate and even contradictory views of the experience. This is because family members feel most supported when their personal experiences are not devalued or ignored.

The key implication for therapists is to help the family develop a shared experience in the healing process without devaluing individual experiences. Our experience was that this process begins to occur during the sharing of stories, when family members identify both the similarities and differences in what they are experiencing. By disclosing feelings and concerns and appreciating each person's unique way of thinking and doing things, family members were able to build greater connections with one another, develop greater empathy within their relationships, find solutions to 
problems in ways that benefited everyone involved, and, ultimately, learn how to cope more effectively with the cancer.

Interactive psychoeducation. Another finding from this study is that interactive psychoeducation was reported by the families to be a critical way for therapists to help families understand their disease and find ways to cope with the stresses it brought into their family system. Family members expressed that one of the main reasons they were coming to therapy was that they really needed to know whether what they were experiencing was normal. As the families made these requests, short, interactive psychoeducational pieces were introduced into the process to provide families with needed information during or after the therapy sessions. Discussing her experience with psychoeducation, Susan stated,

For me personally it helped ... where you talked about what was normal for cancer recovery and that was way helpful to me.... And so to have you come in after having all that emotional session, "Okay, well, here's what cancer recovery looks like, oh, ok, well, you know, now that I know that I'm just fitting in the cycle." Then that relieved a lot of [anxiety]. . . . For me it was the safe way to hear what was normal about cancer.

From this aspect of our work, we conclude that working with families with cancer requires therapists to have at least a general understanding of the common issues that occur in these families. By appropriately discussing this information during the therapy process, therapists can alleviate the common fear of families that they are "doing it wrong" or "not coping correctly." We found that sharing information with the families helped them organize and normalize their experiences.

Putting illness in its place. As discussed earlier, a key therapeutic intervention that was found to be helpful was putting the illness in its place (Gonzalez et al., 1989; Steinglass, 1998). The cancer-dominated new world can be problematic, because cancer begins to rule the family. One strategy used was to help the family and/or individual reclaim unnecessary losses by helping them identify those issues they wanted to "take back from cancer."

Structure and process. The last therapeutic intervention, structuring therapy, was identified as extremely important in working with these families. During the study, three components were found to be important: (a) the benefits of marital sessions, (b) the timing of therapy, and (c) maintaining a here-and-now focus. The benefit of marital sessions is highlighted because issues emerged in the course of therapy (e.g., finances and sexuality) that could not be fully addressed in the family sessions, so there needed to be a balance between family and marital sessions. The second component, timing of therapy, was important because the cancer patient might be physically unable to attend sessions during treatment. The families recommended that the therapy process be divided into two phases: a first phase to provide psychosocial support for the family during the treatments, and a second phase with the patient and family after treatment. Further research needs to identify to what extent the timing of therapy actually affects the outcome. The third component, maintaining a here-and-now focus, is important because families cannot change their past experiences. The family must be able to experiment with changes inspired by the past but practiced in the present.

\section{DISCUSSION}

Overall, we found the processes involved in reflective case analysis to be extremely beneficial in developing some key assessment and intervention strategies for the participant families. Without the direct input of the families, it is evident that these preliminary strategies would not have been as clearly identified. The action 
research process allowed the family members to participate in the therapy sessions and then immediately reflect the effectiveness of the therapy from an observer viewpoint or metaperspective. This evaluation was valuable for two reasons: (a) Each family was able to immediately identify key components of the therapy they viewed as helpful, and (b) these issues were more solidly implanted in their memory, proving to be effective and enduring (Wright et al., 1996).

The limitations of this study are clear. This is an investigatory study devised to shed light on ways therapists and other professionals should work with families dealing with cancer. Because of the study design, the findings cannot be directly generalized to a greater population. However, this study was devised to be generative and produced information about the lived experience of cancer and the process of therapy. We believe that this study can serve as a guiding, preliminary source of information for future studies. Another limitation is the use of families dealing with different forms of cancer, which limits the study's ability to explain unique and similar aspects of different types of cancer. However, the major themes from our analyses emerged from both families and give some indication of common difficulties and successful treatment strategies that may be effective with several forms of cancer. Finally, it should be noted that reflective interviewing procedures are geared toward clients with strong verbal abilities, and further research using creative designs may be needed to asses the experiences of family members who are less articulate or open with their feelings.

Despite its limitations, this study demonstrates that shared experience building is an important component in therapy with families with cancer, and in this sense our findings agree with previous qualitative studies that showed that meaningful discussion among family members improves psychosocial adjustment to cancer (Cooley
\& Moriarty, 1997; Lewis et al., 1993). Additionally, psychoeducation has been found to be an essential part of the treatment for families with cancer, because, often, normalization greatly reduced the anxiety of the participants (Cunningham, 1992; Davis-Ali, Chesler, \& Chesney, 1993; Hilton, 1993).

Successfully coping with cancer is a difficult process that profoundly brings forth the emotional crucible component of family living. This experience can be made easier through the development of a familyshared experience of healing. Additionally, this study demonstrates the unique contribution clients can make in their own therapeutic process while simultaneously informing the therapeutic practices of the therapists who serve them. We believe that implementing action projects with more families is an essential step in contributing to the growth of this type of patient-centered treatment. This type of work provides a unique perspective of this coevolved journey of cancer with families and their collaborative helpers.

\section{REFERENCES}

Baider, L., Cooper, C., \& De-Nour, A. (1996). Cancer and the family. Chicester, England: Wiley.

Becker, E. (1973). The denial of death. New York: Free Press.

Chesler, M., \& Chesney, B. (1995). Cancer and self-help: Bridging the troubled waters of childhood illness. Madison: University of Wisconsin Press.

Chollar, S. (1994). Mind over cancer? American Health, 13, 74-77.

Cohen, M. H. (1993). Diagnostic closure and the spread of uncertainty. Issues in Comprehensive Pediatric Nursing, 16(3), 135-146.

Cooley, M., \& Moriarty, H. (1997). An analysis of empirical studies examining the impact of cancer diagnosis and treatment of an adult on family functioning. Journal of Family Nursing, 3, 318-347.

Cunningham, A. (1992). The healing journey. Toronto, Ontario, Canada: Key Porter Books.

Davis-Ali, S. H., Chesler, M. A., \& Chesney, B. K. (1993). Recognizing cancer as a family disease. Social Work Health Care, 19(2), 4565. 
Galinsky, M. J. (1985). Groups for cancer patients and their families: Purposes and group conditions. In M. Sundel, P. Glasser, R. Sarri, \& R. Venter (Eds.), Individual change through small groups (Vol. 2, pp. 27-42). New York: Free Press.

Gilbert, K. R. (1996). "We've had the same loss, why don't we have the same grief?" Loss and differential grief in families. Death Studies, 20, 269-283.

Gonzalez, S., Steinglass, P., \& Reiss, D. (1989). Putting the illness in its place: Discussion groups for families with chronic medical illness. Family Process, 28, 69-87.

Gosselin, M. (1989). Practice sketches and feedback loops: Giving new meaning to cancer. Family Systems Medicine, 7, 213-218.

Guba, E., \& Lincoln, Y. (1989). Fourth generation evaluation. Newbury Park, CA: Sage.

Halona-Trujillo, M. (1998). Spirituality in a healthcare setting. Hemaware, 3(2), 38-41.

Hilton, B. A. (1993). Issues, problems, and challenges for families coping with breast cancer. Seminars in Oncological Nursing, 9(2), 88100.

Holter, I. M., \& Schwartz-Barcott, D. (1993). Action research: What is it? How has it been used and how can it be used in nursing? Journal of Advanced Nursing, 18, 298-304.

Hull, M. M. (1989). Family needs and supportive nursing behaviors during terminal cancer: A review. Oncology Nursing Forum, 16, 787-792.

Kristjanson, L. J. (1989). Quality of terminal care: Salient indicators identified by families. Journal of Palliative Care, 5(1), 21-30.

Kristjanson, L. J., \& Ashcroft, T. (1994). The family's cancer journey: A literature review. Cancer Nursing, 17(1), 1-17.

Levinson, W., \& Roter, D. (1995). Physicians' psychosocial beliefs correlate with their patient communication skills. Journal of General Internal Medicine, 10, 375-379.

Lewis, F. M., Hammond, M. A., \& Woods, N. F. (1993). The family's functioning with newly diagnosed breast cancer in the mother: The development of an explanatory model. Journal of Behavioral Medicine, 16, 351-370.

Lindemann, E. (1944). Symptomatology and management of acute grief. American Journal of Psychiatry, 101, 141-148.

McDaniel, S., Hepworth, J., \& Doherty, W. (1997). The shared experience of illness. New York: Basic Books.

Norman, A., \& Brandeis, L. (1992). Adddressing the needs of survivors: An action research approach. Journal of Psychosocial Oncology, 10(1), 3-18.
Northhouse, L. (1996). Sharing the cancer experience: Husbands of women with initial and recurrent breast cancer. In A. De-Nour (Ed.), Cancer in the family (pp. 305-320). Chichester, England: Wiley.

Ong, L. M. L., Visser, M. R. M., Lammes, F. B., \& Haes, J. C. J. M. (2000). Doctor-patient communication and cancer patients' quality of life and satisfaction. Patient Education and Counseling, 41, 145-156.

Ostroff, J., \& Steinglass, P. (1996). Psychosocial adaptation following treatment: A family systems perspective on childhood cancer survivorship. In L. Baider, C. Cooper, \& A. DeNour (Eds.), Cancer in the family (pp. 129148). Chichester, England: Wiley.

Pitceathly, C., \& Maguire, P. (2003). The psychological impact of cancer on patients' partners and other key relatives: A review. European Journal of Cancer, 39, 1517-1524.

Reason, P. (1994). Participation in human inquiry. Thousand Oaks, CA: Sage.

Reason, P. (1996, September). Action research. Paper presented at the Qualitative Health Research Conference, Bournemouth, England.

Reele, B. L. (1994). Effect of counseling on quality of life for individuals with cancer and their families. Cancer Nursing, 17(2), 101112.

Robinson, W. D., \& Smith, C. (1998). Understanding hemophilia: A family process of adaptation. Unpublished manuscript.

Rolfe, G. (1996). Going to extremes: Action research, grounded practice and the theorypractice gap in nursing. Journal of Advanced Nursing, 24, 1315-1320.

Rolland, J. (1994). Families, illness, \& disability. New York: Basic Books.

Scott, J., Prictor, M., Harmsen, M., Broom, A., Entwistle, V., Sowden, A., et al. (2003). Interventions for improving communication with children and adolescents about a family member's cancer. Cochrane Database Systems Review, 4, CD004511.

Seaburn, D., Lorenz, A., Gunn, W., Gawinski, B., \& Mauksch, L. (1996). Models of collaboration: A guide for mental health professionals working with health care practitioners. New York: Basic Books.

Sellers, T. S. (2000). A model of collaborative healthcare in outpatient medical oncology. Families, Systems \& Health, 18, 19-33.

Shuman, R. (1996). The psychology of chronic illness. New York: Basic Books.

Spiegel, D. (1990). Can psychotherapy prolong cancer survival? Psychosomatics, 31, 361-366. 
Spiegel, D., Bloom, J. R., Kraemer, H. C., \& Gottheil, E. (1989, October 14). Effect of psychosocial treatment on survival of patients with metastatic breast cancer. Lancet, 2, $888-891$.

Stedeford, A. (1981). Couples facing death: Unsatisfactory communication. Cancer Nursing, 13, 234-245.

Steinglass, P. (1998). Multiple family discussion groups for patients with chronic medical illness. Families, Systems \& Health, 16, 55-70.

Strauss, A., \& Corbin, J. (1998). Basics of qualitative research: Techniques and procedures for developing grounded theory (2nd ed.). Thousand Oaks, CA: Sage.

Temoshok, L., \& Dreher, H. (1992). The Type $C$ connection: The behavioral links to cancer and your health. New York: Random House.

Wamboldt, F. S., \& Reiss, D. (1989). Defining a family heritage and a new relationship identity: Two central tasks in the making of a marriage. Family Process, 28, 317-335.

Wright, L., Watson, W., \& Bell, J. (1996). Beliefs: The heart of healing in families and illness. New York: Basic Books. 
Copyright of Families, Systems \& Health: The Journal of Collaborative Family HealthCare is the property of American Psychological Association. The copyright in an individual article may be maintained by the author in certain cases. Content may not be copied or emailed to multiple sites or posted to a listserv without the copyright holder's express written permission. However, users may print, download, or email articles for individual use. 